\title{
Are cutout handles used when available in real occupational settings? Description of grips and upper extremities movements during industrial box handling
}

\author{
Luciana C. C. B. Silva ${ }^{\text {a, }}$, Ana B. Oliveira ${ }^{a}$, Michele E. R. Alem ${ }^{a}$, Luis C. Paschoarelli ${ }^{b}$ and Helenice J. \\ C. G. Coury ${ }^{\mathrm{a}}$. \\ ${ }^{a}$ Department of Physical Therapy, Federal University of São Carlos, Rodovia Washington Luis km 235, \\ Monjolinho, 13565-905, São Carlos, SP, Brazil \\ bDepartment of Industrial Design, Faculty of Architecture, Arts and Communication, São Paulo State University, \\ Av. Eng. Luiz Edmundo C. Coube, 14-01 17033-360 Bauru, SP, Brazil
}

\begin{abstract}
In order to achieve better postures and decrease musculoskeletal risks adequate design of hand/box couplings for manual materials handling $(\mathrm{MMH})$ are still needed. No studies evaluating upper limb movement thorough direct measurements during box handling in workplace were identified in the literature. In this study we describe the types of grip and movements adopted by ten workers when handling redesigned boxes with cutout handles between different heights on industrial pallets. The new handles were used by $90 \%$ of the workers through different types of grip. Electrogoniometric measurements showed relatively safe forearm and wrist movements, although elbow inadequate range of movement was recorded. Despite the good acceptance of the cutout by workers, the new design requires extra internal space in the boxes reducing applications for this alternative of box.
\end{abstract}

Keywords: industrial handling, movement recording, electrogoniometer, work related musculoskeletal disorders, wrist

\section{Introduction}

Despite the intense mechanization and rapid technological advances, manual materials handling $(\mathrm{MMH})$ is still common in occupational environments and represents a major occupational safety concern in industry [8]. Studies have evaluated the lower back during MMH extensively whilst upper limbs, which are the second body part most affected by musculoskeletal disorders during this task [5], remain under evaluated so far. Forceful movements and large wrist amplitudes have been associated with workload and musculoskeletal disorders [12]. Wrist deviations from the neutral position may increase the pressure in the carpal tunnel and decrease the lever arm for intrinsic muscles, increasing musculoskeletal load and the risk of these disorders [14].

In this context, the use of box handles has been accepted as a means of improving the hand/box coupling, in order to avoid extreme range of movements and increase the maximum acceptable weight of carriage [4]. However, very few boxes have handles and, when available, they were not always used [6]. Thus, it can be hypothesized that if handles were designed following ergonomic principles and supported by participative approach, the results could be different.

Cardboards boxes with cutout handles, designed after a participative intervention in an industrial packing and stoking sector, were evaluated in this study. The design of the handles was suggested by

*Corresponding author. E-mail: lucianaccbs@yahoo.com.br. 
workers, and after the analysis of its viability, one of the designs was adopted by the company for inside transportation of materials. It was expected that the handles would be used by the workers and promote safer upper extremities movements. Thus, the objective of this study were to: 1) describe types of grip adopted while handling the new boxes with cutout handles and; 2) evaluate the amplitudes of upper limb movements (wrist flexion/extension, ulnar/radial deviations, forearm pronation and supination and elbow flexion), recorded by electrogoniometers when workers performed palletizing and depalletizing tasks in a real industrial setting using the redesigned boxes.

\section{Methods}

Ten male right handed subjects (mean age $38.2 \pm$ 8.3 years, mean weight $78.3 \pm 13.2 \mathrm{~kg}$ and mean height $1.7 \pm 0.05 \mathrm{~m}$ ) took part in the study. These subjects had at least one year of previous experience on pallet tasks. The workers were non-symptomatic for work related musculoskeletal disorders during the period of data collection. The study was approved by the local ethics committee (CAAE 0054.0.135.00007).

The boxes were used for inside transportation of empty packaging from the printing division (in which empty packing, blisters etc were manufactured) to the production lines (where the empty packagings were filled in with products). The handling task consisted of piling up cardboard boxes on a pallet. The workers could fold the flaps either before or after each handling. The pallet was then mechanically moved to another sector where the depalletizing task occurred. The complete palletizing and depalletizing tasks involved piling up and down 16 boxes, distributed in four layers, totalizing $16 \mathrm{MMHs}$. The cardboard boxes dimensions were $53 \mathrm{~cm}$ long, $47 \mathrm{~cm}$ wide and 27 $\mathrm{cm}$ high and the lateral cutouts consisted of oval orifices of dimensions $9 \times 4 \mathrm{~cm}$. The box was designed big enough to provide some extra clearance for the workers fingers to be inserted in the holes (cutouts) of the box. The structure of cardboard was stronger to allow for several reutilizations of box, potentializing it's use. The box weight ranged from 7 to $20 \mathrm{~kg}$. The workers were familiarized with the redesigned box as it had been used for six months before the data collection.

Bilateral upper limb movements were recorded using an electrogoniometers sensors (model XM65 for wrist: flexion-extension and radial-ulnar deviation;
XM110 for elbows: flexion-extension; and Z110 for forearms: pronation-supination) and a DataLog acquisition unit [3]. These electrogoniometers had previously been evaluated and considered to be reliable and accurate [13]. The sampling frequency was set at $100 \mathrm{~Hz}$.

\section{Procedures}

Electrogoniometers were attached to the subjects using double-side tape. The electrogoniometers were mounted and calibrated in accordance with the manufacturer's instructions [3]. Subjects were instructed to perform their routine work naturally.

Data were recorded throughout the whole tasks. Besides the handling tasks the workers were responsible for controlling the packing machine. For identifying the $\mathrm{MMH}$ period, the whole work cycle was filmed and the video was synchronized with the electrogoniometer recording. This procedure allowed for the precise identification of the handling tasks. As the boxes handling were performed in between the same heights, results from both tasks (palletizing/depalletizing) were analyzed altogether.

\section{Data Analysis}

The types of grip adopted during the handling activities were evaluated descriptively. The electrogoniometer data were reduced through a MatLab (7.0.1, The Mathworks Inc., Natick, MA, USA) routine. The data were low-pass filtered at $5 \mathrm{~Hz}$ using a secondorder zero-lag Butterworth filter. The cut-off frequency was determined from a residual analysis. The $10^{\text {th }}, 50^{\text {th }}$ and $90^{\text {th }}$ percentiles of the ROMs for each of the handling tasks were calculated for each subject.

The presence of upper limb postural risks was evaluated according to safe ROM described in previous reviews available in the literature [11,9]. Wrist movements within $\pm 15^{\circ}$ of flexion $(+)$ /extension (-) and $10^{\circ}$ radial $(-) / 15^{\circ}$ ulnar $(+)$ deviation and amplitudes of up to $45^{\circ}$ for pronation/supination movements were considered to be safe as these ranges present biomechanical advantage [11]. For the elbow joint, the flexion range from $80^{\circ}$ to $120^{\circ}$ was considered adequate, since this includes the ROM of greatest biomechanical advantage for the main muscles involved in elbow flexion [9]. 


\section{Results}

Five different types of grip were observed during the handling tasks (Figure 1 - left side). The grips were described in terms of whether the cutout handles of the box were used or not: Grip A - both handles were used; Grip B.1 - only one hand (the right) was inserted at the cutout, while the other (left hand) was on the lower edge of the contralateral flap of the box (not illustrated); Grip B.2 - the opposite of the preceding situation: only one hand (the left) was inserted at the cutout, while the other (right hand) was on the lower edge of the contralateral flap of the box; Grip C - left hand inserted at the cutout and the right hand at the bottom of the box; Grip D - cutouts were not used, the edges of the sides of the box were used to hold it during the handling task.

The majority of the workers $(90 \%)$ used the cutouts, either symmetrically or asymmetrically (Figure 1 A, B.1, B.2 and C). Different types of the grips were adopted by the workers, however, the grip chosen for each one of them tended to be kept constant during the whole handling task. Only one of the subjects presented two types of grip (Grips A and B.2). The number of workers adopting each type of grip was: Grip A - 4 workers; Grip B.1 - 1 worker; Grip B.2 2 workers; Grip C - 1 worker; Grip D - 1 worker; and 1 worker for the combination of two grips (Grips A and B.2). Regarding symmetry, about $50 \%$ of the workers grips can be considered symmetrical (Grip A and D).
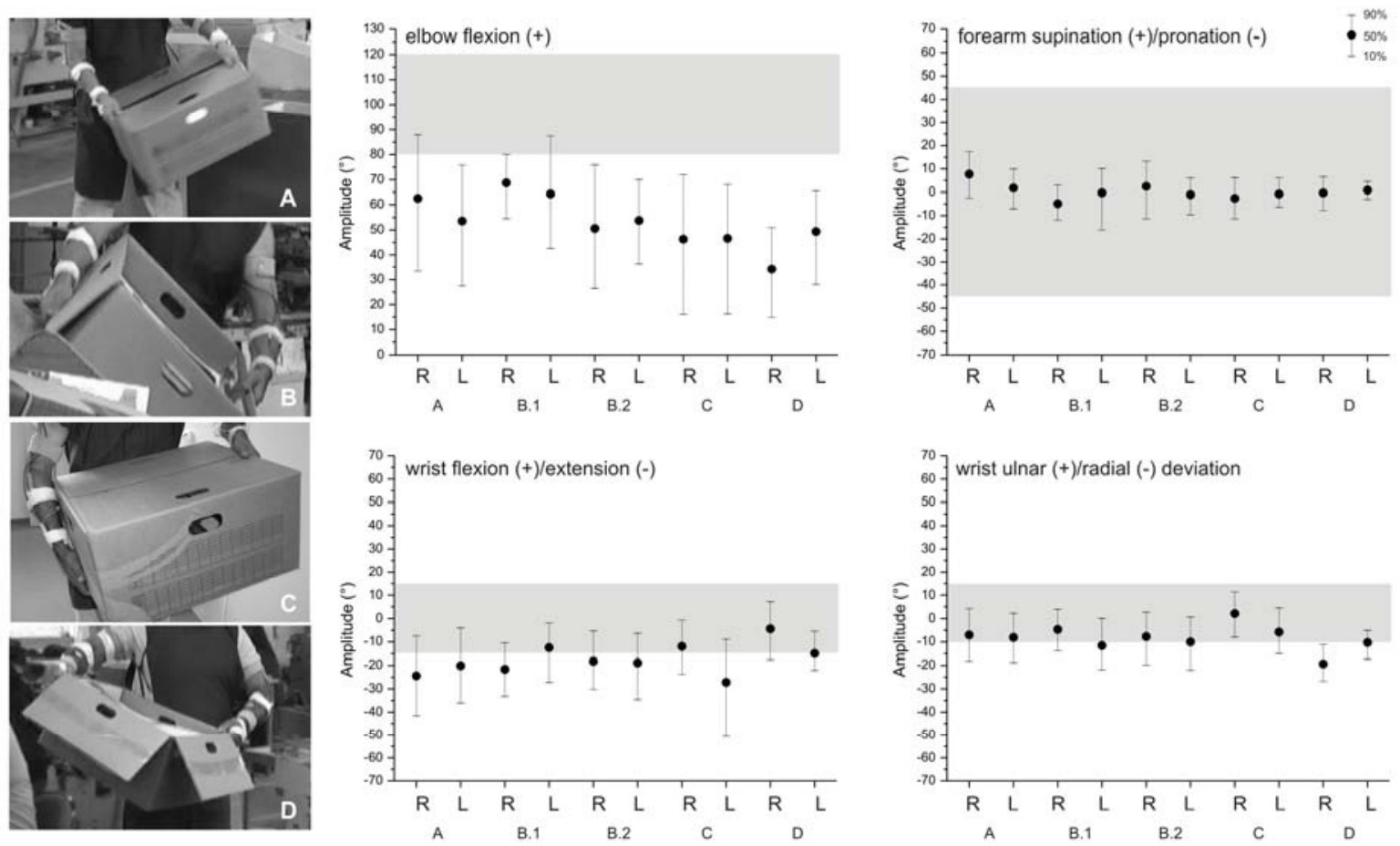

Figure 1: Left side: Grip types adopted - A: both hands using the handles of the box; B: one hand using the handle and the other the flaps of the box; $\mathrm{C}$ : left hand using the handle and the right at the bottom of the box and; D: both hands gripping the upper edges of the box. Right side: The mean values of $10^{\text {th }}$ percentile ()$, 50^{\text {th }}$ percentile $(\bullet)$ and $90^{\text {th }}$ percentile ( ) of each type of grip for, respectively, the right $(\mathrm{R})$ and left $(\mathrm{L})$ elbow flexion-extension, forearm pronation-supination, wrist flexion-extension, and wrist radial-ulnar deviation. The grey band represents the safe ROM for each movement. 
The mean values of $10^{\text {th }}, 50^{\text {th }}$ and $90^{\text {th }}$ percentiles of the arm movements during the handling tasks are presented in Figure 1 (right side) for each grip type described previously. The wide range between $10^{\text {th }}$ and $90^{\text {th }}$ percentile values indicates high variability between subjects for all conditions, but particularly for elbow and wrist movements.

The $50^{\text {th }}$ percentile values for elbow ROM were outside of the safe range for all grip types (Figure 1elbow flexion). The $50^{\text {th }}$ percentile value that was furthest from the safe range was identified for the right elbow during grip D. All $50^{\text {th }}$ percentile values of the pronation/supination movement were recorded within the safe ROM (Figure 1-forearm supination/pronation).

\section{Discussion}

The most frequent movements recorded for both the right and the left limbs were extension and radial deviation (Figure 1-wrist flexion/extension and wrist ulnar/radial deviation). The $50^{\text {th }}$ percentiles values of wrist flexion/extension were outside of safe limit for most of the grip types. The $50^{\text {th }}$ percentile value furthest from the safe range was identified for the left wrist with grip C (Figure 1-wrist flexion/extension). For most of the grips, the $50^{\text {th }}$ percentile values of radial/ulnar deviations remained at the lower limit of the safe range (Figure 1-wrist ulnar/radial deviation). The furthest $50^{\text {th }}$ percentile value from the safe range was found for the right wrist with grip D.

If the handles were used symmetrically more homogenous movements among workers could have been achieved. Nevertheless, results showed a high variability of grip types, and a low variability within workers. In this sense, the variation within individual can be considered very low, as the majority of the workers (90\%) have adopted a unique type of grip during the whole tasks.

The worker preference for one type of grip can be explained by their long experience on the job, i.e., at least one year of previous experience on pallet tasks. Experienced workers tend to develop individual strategies that can improve the balance and permit better control during load transferring [2]. These strategies, not always symmetrical, are developed by each work to enhance the biomechanical advantage of the handling. In the present study, observation of the video recording of the tasks indicated that the box tended to be laterally tilted. In three types of grips (grips B.1, B.2 and C) the box was also held by its bottom. The use of the bottom surface for hand coupling was reported mainly when heavier boxes (10 $\mathrm{kg}$ ) were handled [10]. In similar way, the preference for upper grip were showed when the box evaluated were lighter $(4.2 \mathrm{~kg})$ [7]. Choosing to handle heavier boxes from its bottom allows for the box center of mass being brought closer to the worker's body. Moreover, the weight distribution of the box between fingers and palm enhances more control over the handling. Altogether, these different strategies seem to minimize the stress at upper limb joints and low back during handling and should be considered when new boxes are designed.

Despite the differences in types of grip adopted in the present study, the number of workers using the cutout was high, particularly considering that they did not have any training for using it. The fact that most of the workers were still using the cutouts after months that they were introduced seems to be a clear indicative of the acceptance of this alternative. The fact that the design was proposed after a participative intervention might also have played a role in these results. Despite that, a problem in this design was also observed. The use of cutouts requires some clearance for the fingers to be inserted in the box, and as a consequence, the box is a bit larger than the necessary space for accommodating the materials. Considering that this extra space can have effect in the products to be transported and also requires more room in the stoking areas, this can be a considerable disadvantage. Thus, this design can be useful only for particular box applications, such as, inside transportation. On the other hand, the boxes can be reutilized and become a substitute for plastic boxes with ecological advantage.

No previous study has objectively compared box with and without cutouts in real occupational situations through direct measurements. In a laboratory study, Ando et al. [1] have compared strength and perceived exertion in three conditions of box lifting (grasping the box from its bottom, from upper and lower corners of the box obliquely, and through a rectangular cutout near the upper edges of the box). The results showed that the cutout conditions was considered to be the best one among the three possibilities evaluated. In the present study the wrist and elbow movements were objectively evaluated in real occupational setting, however, no data is available from the older boxes (without cutouts) used in the company to compare boxes with and without cutout. Regarding general types of handles, it has long been recognized that the use of handles is advantageous to reduce extreme range of movements and increase the 
maximum acceptable weight of handling [4], suggesting that cutout boxes would also be comparatively favorable in comparison with boxes without handles.

Although the sample size of the study can be considered relatively small, it has comprised the whole team of employees who performed $\mathrm{MMH}$ activities in the packing and stocking sector of an industrial company. Furthermore, these workers were submitted to the same conditions and work requirements which make the sample and conditions less influenced by other sources of variation.

Considering the lack of studies evaluating upper limbs during manual handling tasks in real situations, and the fact that the present study has identified limitations for the box design evaluated here, new studies should be performed in order to evaluate alternative box designs in occupational situations. Moreover, the recording of other joints, such as, shoulders and back should be associated with the evaluation of upper extremities in order to improve the understanding on exposure, thereby enabling safer postures for the hands and arms during MMH tasks

This study was partially supported by $\mathrm{CNPq}$ and Fapesp.

\section{References}

[1] S. Ando, Y. Ono, M. Shimaoka, S. Hiruta, Y. Hattori, F. Hori, T. Imaeda, Y. Takeuchi, Strength and perceived exertion in isometric and dynamic lifting with three different hand locations, Journal of Occupational Health 42 (2000), 315-20.
[2] M. Authier, M. Lortie, M. Gagnon, Manual handling techniques:Comparing novices and experts, International Journal of Industrial Ergonomics 17 (1996), 419-29.

[3] Biometrics, Goniometer and torsiometer operating manual Gwent, United Kingdom, 2007.

[4] T-S. Cheng, T-H. Lee, Maximum acceptable weight of manual load carriage for young Taiwanese males, Industrial Health 44 (2006), 200-06.

[5] P.G. Dempsey, L. Hashemi, Analysis of workers' compensation claims associated with manual material handling, Ergonomics 42 (1999), 183-95.

[6] C.G. Drury, C.H. Law, C.S. Pawensky, A survey of industrial box handling, Human Factors 24 (1982), 553-65.

[7] H-S. Jung, H-S. Jung, A survey of the optimal handle position for boxes with different sizes and manual handling positions, Applied Ergonomics 41 (2010), 115-22.

[8] C. J. Lin, S.J. Wang, H.J. Chen, A field evaluation method for assessing whole body biomechanical joint stress in manual lifting tasks, Industrial Health 44 (2006), 604-12.

[9] W.M. Murray, S.L. Delp, T.S. Buchanan, Variation of muscle moment arms with elbow and forearm position, Journal of Biomechanics 28 (1995), 513-25.

[10] R.S. Padula, V.C. Souza, H.J.C. Gil Coury, Types of grip and wrist movements during load handling activities, Brazilian Journal of Physical Therapy 10 (2006), 29-34.

[11] L.C. Paschoarelli, A.B. Oliveira, H.J.C. Gil Coury, Assessment of the ergonomic design of diagnostic ultrasound transducers through wrist movements and subjective evaluation, International Journal of Industrial Ergonomics 38 (2008), 9991006.

[12] L.P. Sande, H.J.C.G. Coury, J. Oishi, S. Kumar, Effect of musculoskeletal disorders on pretensions strength, Applied Ergonomics 32 (2001), 609-16.

[13] A. Shiratsu, H.J.C.G. Coury, Reability and accuracy of different sensors of a flexible eletrogoniometer, Clinical Biomechanics 8 (2003), 682-84.

[14] R. Wells, P. Keir, Work and Activity-related Musculoskeletal Disorders of the Upper Extremity in Biomechanics in Ergonomics, Ed by S Kumar, Taylor \& Francis, London, 1998. 